\title{
SPECTROSCOPICAL STUDY OF FAINT SOUTHERN CEPHEIDS WITH SOUTHERN AFRICAN LARGE TELESCOPE (SALT). FIRST RESULTS.
}

\author{
I. A. Usenko ${ }^{1,2}$, A. Yu. Kniazev ${ }^{3,4}$, V. V. Kovtyukh' ${ }^{1}$, S. I. Belik ${ }^{1}$ and L. N. Berdnikov ${ }^{5}$ \\ ${ }^{1}$ Astronomical Observatory, Odessa National University, Marazlievska 1B, \\ Odessa 65014, Ukraine,vkovtyukh@ukr.net \\ 2 Mykolaiv Astronomical Observatory, Obsevatorna 1, \\ Mykolaiv 54030, Ukraine, igus99@ukr.net \\ 3 South African Astronomical Observatory, P.O. 7925, \\ Cape Town, South Africa akniazev@saao.ac.za \\ ${ }^{4}$ Southern African Large Telescope Foundation, P.O. 7925, \\ Cape Town, South Africa \\ 5 Sternberg Astronomical Institute, Universitetskii pr. 13, \\ Moscow 119991, Russia lberdnikov@yandex.ru
}

\begin{abstract}
First Cepheids observations using echelle-spectrograph HRS fed by Southern African Large Telescope (SALT) were realized during 2016. All spectra have been obtained in the medium resoltion mode $(\mathrm{R} \approx 31000-41000)$ wth high $\mathrm{S} / \mathrm{N}$ ratio near 50-220. All data were processed using package developed by authors based on the standard system of astronomical data reduction MIDAS. Using new echelle data we found the atmosphere parameters and chemical composition for 30 faint Cepheids of southern hemisphere, where for the most of theses stars these results we obtained for the first time. 28 stars are Cepheids after the first-dredge up stage, while ASAS 075842-25336.1 and ASAS 1131714-6605.0 having remarkable Li I $6707.8 \AA$ absorption line and anomalous $\mathrm{CNO}$ and $\mathrm{Na}$ content could be consider as first crossing of the Cepheids instability strip.
\end{abstract}

Keywords: Stars: Cepheids: atmosphere parameters; Cepheids: chemical composition

\section{Introduction}

The abundance gradient for the Galaxy as observational characteristic of the galactic disk is the most input parameter in any theory of galactic chemical evolution. Many questions concerning the present-day abundance distribution in the galactic disk, its spatial properties and evolution with time remain to be answered. To answer these questions it would be reasonably to use the Cepheids as the quite suitable probes of metallicity in the Galactic disc.

According to results of investigations for iron, for example, its abundance gradient displays a multimodal structure: a rather flat part in vicinity of the Sun, a small gradient in the outer part of the disk (here the distribution shows some scatter and all the stars are metal-deficient comparing to the stars from the solar vicinity), and a quite large negative gradient in the inner part of the disk in the range from 4 to $7 \mathrm{kpc}$, but it with a very small number of investigated stars, and poorer statistics.

Even though elemental abundance increases towards the galactic center, there are arise two questions: 1) what is the real behavior of the abundance distributions within the inner parts (less than $7 \mathrm{kpc}$ ) of the galactic disk; 2) what is the real one for the galactocentric distances more than $10 \mathrm{kpc}$ ?

Therefore, the main objective of our program is to observe additional Cepheids situated closer to the galactic center and situated too much far away from it in order to:

1) Constrain the metallicity distribution and its gradient in these regions;

2) Find the properties of the abundance distribution at galactocentric distances of less than $7 \mathrm{kpc}$ and more than $10 \mathrm{kpc}$.

3) To extract the possible objects belonging to the Population II.

To realize this program we have used Southern African Large Telescope (SALT). Our observational 
Table 1: List of investigated Cepheids.

\begin{tabular}{|c|c|c|c|c|c|c|c|c|c|c|}
\hline Object & Type & $\mathrm{P}(\mathrm{d})$ & $\mathrm{R}_{G}(\mathrm{kpc})$ & {$[\mathrm{Fe} / \mathrm{H}]$} & {$[\mathrm{C} / \mathrm{H}]$} & {$[\mathrm{N} / \mathrm{H}]$} & {$[\mathrm{O} / \mathrm{H}]$} & {$[\mathrm{Na} / \mathrm{H}]$} & {$[\mathrm{Mg} / \mathrm{H}]$} & {$[\mathrm{Al} / \mathrm{H}]$} \\
\hline V1048 Cen & DCEP & 1.300 & 7.44 & -0.09 & -0.35 & +0.38 & -0.35 & +0.15 & -0.12 & +0.10 \\
\hline ASAS 114920-6600.6 & DCEPS(B) & 1.960 & 7.32 & -0.04 & -0.20 & +0.34 & +0.52 & +0.12 & -0.21 & +0.15 \\
\hline ASAS 072424-0751.3 & DCEPS & 2.071 & 9.76 & -0.19 & -0.43 & +0.22 & - & +0.25 & +0.18 & +0.02 \\
\hline ASAS 073200-2529.3 & DCEPS & 2.421 & 9.25 & -0.20 & -0.47 & +0.14 & +0.05 & +0.11 & -0.20 & -0.04 \\
\hline ASAS $174603-3528.1$ & DCEPS & 2.573 & 6.02 & +0.11 & +0.04 & +0.60 & - & +0.41 & -0.15 & +0.43 \\
\hline ASAS 183652-0907.1 & DCEP & 2.590 & 6.55 & +0.14 & -0.21 & +0.42 & -0.15 & +0.45 & +0.16 & +0.33 \\
\hline BD-10 4739 & DCEP & 3.058 & 6.63 & +0.04 & -0.24 & +0.28 & -0.29 & +0.30 & -0.18 & +0.25 \\
\hline 104130-5956.9 & DCEPS & 3.081 & 7.56 & +0.00 & -0.26 & +0.39 & +0.15 & +0.18 & -0.06 & +0.18 \\
\hline V720 Car & DCEP & 3.081 & 7.56 & -0.03 & -0.27 & +0.36 & -0.28 & +0.17 & -0.06 & +0.13 \\
\hline ASAS $065851-1344.2$ & DCEPS & 3.280 & 11.55 & -0.29 & -0.59 & +0.18 & -0.35 & +0.06 & -0.24 & -0.09 \\
\hline FZ Car & DCEP & 3.578 & 7.64 & +0.05 & -0.21 & +0.51 & -0.28 & +0.29 & +0.01 & +0.21 \\
\hline HD 317966 & DCEP & 3.720 & 6.90 & +0.08 & -0.15 & +0.64 & -0.23 & +0.40 & +0.10 & +0.23 \\
\hline ASAS 100814-5856.6 & DCEPS & 3.767 & 7.78 & -0.07 & -0.36 & +0.24 & -0.35 & +0.11 & +0.02 & +0.16 \\
\hline HD 160473 & DCEP & 3.780 & 6.81 & +0.04 & -0.20 & +0.51 & -0.11 & +0.23 & +0.10 & +0.21 \\
\hline V701 Car & DCEP & 4.090 & 7.71 & -0.01 & -0.21 & +0.51 & - & +0.43 & +0.00 & +0.22 \\
\hline V690 Car & DCEP & 4.150 & 7.85 & +0.12 & -0.18 & +0.46 & -0.04 & +0.38 & +0.04 & +0.32 \\
\hline V1210 Cen & DCEP & 4.320 & 6.88 & +0.08 & -0.08 & +0.16 & -0.25 & +0.15 & +0.39 & +0.32 \\
\hline GI Car & DCEP & 4.431 & 7.48 & -0.04 & -0.33 & +0.34 & -0.31 & +0.18 & -0.09 & +0.13 \\
\hline CC Car & DCEP & 4.760 & 7.76 & +0.09 & -0.17 & +0.47 & -0.28 & +0.30 & -0.06 & +0.33 \\
\hline ASAS $182714-1507.1$ & DCEP & 5.550 & 6.02 & +0.32 & -0.01 & +0.90 & -0.26 & +0.67 & +0.23 & +0.58 \\
\hline ASAS $123617-6317.6$ & DCEPS & 6.166 & 6.86 & +0.09 & -0.21 & +0.47 & - & +0.34 & +0.22 & +0.32 \\
\hline RS Nor & DCEP & 6.198 & 6.26 & +0.15 & -0.11 & +0.60 & +0.32 & +0.54 & +0.11 & +0.37 \\
\hline ASAS 070832-1454.5 & DCEPS & 6.388 & 9.58 & -0.06 & -0.38 & +0.26 & -0.52 & +0.15 & -0.26 & +0.11 \\
\hline ASAS 092758-5218.9 & DCEP & 7.640 & 8.33 & +0.05 & -0.19 & +0.35 & - & +0.35 & +0.22 & +0.26 \\
\hline ASAS 093942-4931.5 & DCEPS & 7.754 & 8.08 & -0.01 & -0.24 & +0.43 & -0.28 & +0.18 & -0.00 & +0.18 \\
\hline VX Cru & DCEP & 12.213 & 6.78 & +0.24 & -0.03 & +0.67 & -0.09 & +0.68 & +0.25 & +0.46 \\
\hline ASAS 083611-3903.7 & DCEP & 12.960 & 8.39 & -0.08 & -0.37 & +0.13 & -0.09 & +0.10 & -0.03 & +0.08 \\
\hline VW Cen & DCEP & 15.036 & 6.28 & +0.38 & +0.15 & +0.74 & -0.19 & +0.67 & +0.18 & +0.53 \\
\hline ASAS 075842-2536.1 & DCEPS(B) & 0.580 & 9.03 & -0.17 & -0.30 & -0.14 & -0.06 & -0.14 & -0.46 & +0.06 \\
\hline ASAS $131714-6605.0$ & DCEPS & 1.290 & 6.85 & +0.05 & -0.17 & +0.68 & +0.01 & +0.36 & -0.05 & +0.40 \\
\hline
\end{tabular}

list contains 168 Cepheids located at galactocentric distances smaller than $7 \mathrm{kpc}$ and more than $10 \mathrm{kpc}$ (Mel'nik et al., 2015). They are to be bright enough to be accessible with SALT.

\section{Observations, data reducing and objects} selection

All observations have been taken using 11m SALT (Southern African Large Telescope) equipped by HRS (High Resolution Spectrograph). HRS is a dual-beam (3700-5500 \& 5500-8900 Å) fiber-fed, white-pupil, echelle-spectrograph, employing VHP gratings as cross dispersers. We obtained even one spectrum for each Cepheid using medium mode spectral resolution $(\mathrm{R}=$ 40000), mean $\mathrm{S} / \mathrm{N}=100$ and more, and it could be enough to resolve our observational tasks. These spectra will be used to obtain the atmosphere parameters and chemical composition of these unexplored yellow supergiants. The data were reduced using the échelle package which consist of huge amount of basic procedures for echelle data reduction. FEROS is another package and it was developed for the reduction of of echelle data from Fiber-fed Extended Range Optical Spectrograph (FEROS). FEROS looks very similar to HRS and both instruments have very close type of echelle data.

DECH30 package (Galazutdinov, 2007) allows to measure the line depths and radial velocities using spectra in FITS format. Lines depths were used to determine the effective temperature (a method based on the spectroscopic criteria, Kovtyukh, 2007).
78 objects with magnitude $10^{m} \leq \mathrm{V} \leq 12 .^{m} 5$ have been selected from GCVS, ASAS and 2MASS catalogues. At now we have the results for spectra of 30 Cepheids. All these data are given in Table 1.

\section{Atmosphere parameters and chemical composition}

\subsection{Methods}

The effective temperatures $\mathrm{T}_{\text {eff }}$ were determined by a method based on the depth ratios of selected pairs of spectral lines most sensitive to the temperature. Several spectroscopic criteria (Kovtyukh, 2007) were used in this case. This method provide an internal accuracy of $10-30 \mathrm{~K}$ for $\mathrm{T}_{\text {eff }}$ (the error of the mean). The microturbulent velocity $\mathrm{V}_{t}$ was determined from the condition for the Fe I abundance derived from a set of lines being independent of their equivalent widths. The surface gravity $\log g$ was determined from the ionization equilibrium condition for Fe I and Fe II atoms.

When estimating the atmospheric parameters and chemical abundances, we used the VALD oscillator strengths (Kupka et al., 1999) and model atmospheres from Castelli \& Kurucz (2004).

\section{2. $R S$ Nor as a testing object}

We used RS Nor as a testing object since its atmosphere parameters and chemical composition were determined earlier by Luck (2014). Our carbon and oxygen abundances estimations are close to ones from Luck (2014) paper, thereas sodium and iron show 
some less values $([\mathrm{Na} / \mathrm{H}]=+0.77$ and $[\mathrm{Fe} / \mathrm{H}]=+0.23$ from Luck paper). All our data are given in Table 1.

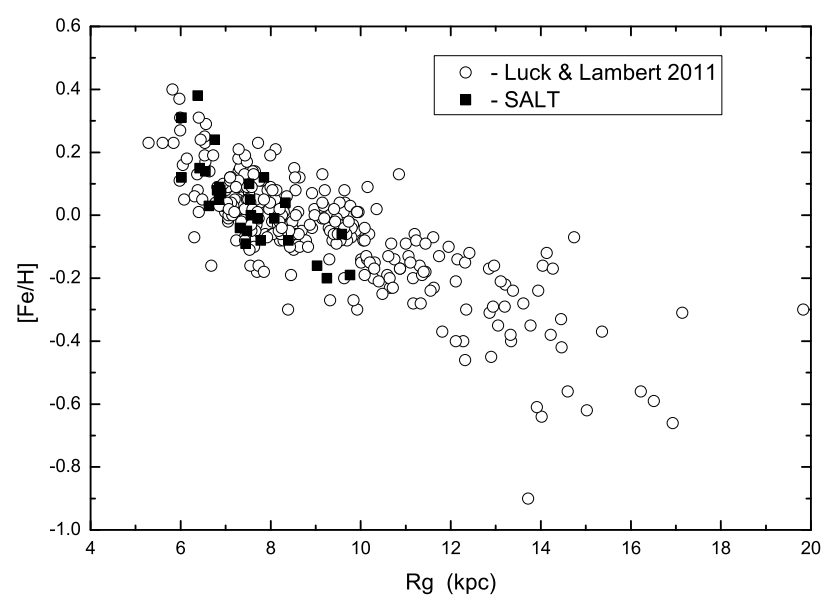

Figure 1: Metallicity gradient of Galaxy according to Cepheids $[\mathrm{Fe} / \mathrm{H}]$ values. Open circles - Luck \& Lambert (2011), filled squares - SALT data.

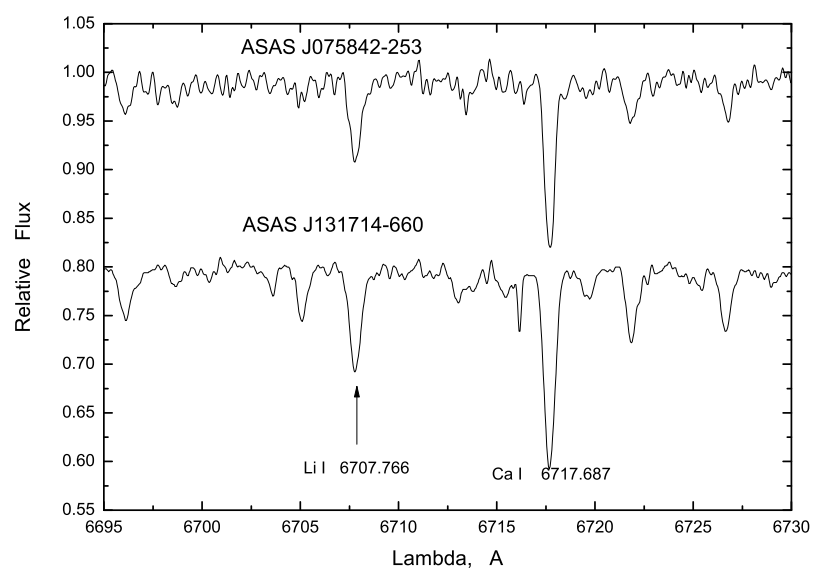

Figure 2: Spectral region around the $6707.8 \AA \mathrm{Li}$ I line in the spectra of ASAS 075842-2536.1 and ASAS 131714-6605.0.

\section{Conclusions}

Our analysis of the available spectra results to the following conclusions:

1. As seen from Table 1 all objects have metallicity values within the ranges of $-0.3-+0.4$ dex that is an evidence of their belonging to Population I.

2. Our $[\mathrm{Fe} / \mathrm{H}]$ estimates for these Cepheids are in good agreement with Luck \& Lambert (2011) data for iron abundance gradient of Galaxy.

3. CNO- elements abundances in the whole show the typical values for yellow supergiants after first dredge-up stage. Carbon is in deficient, excepting ASAS 174603-3528.1, ASAS 182714-1507.1 and VW Cen. These Cepheids have metallicity overabundance and their $[\mathrm{C} / \mathrm{Fe}]$ relation give the deficient too. Nitrogen demoinstrates overbundance except of case for ASAS 075842-2536.1. There are significant discrepancies of oxygen from the solar value.

4. Sodium is in overabundace, exept ASAS 0758422536.1. Magnesium abundance shows discrepances and aluminium content is overabundant in most cases except for ASAS 073200-2529.3 and ASAS 065851-1344.2

5. Objects ASAS 075842-2536.1 and ASAS 1317146605.0 have remarkable absorption line of lithium $6707.8 \AA$ (see Figure 2), and anomalous CNO and $\mathrm{Na}$ content. Quite probably these stars are Cepheids, first time crossing the Cepheids instability strip (CIS).

\section{References}

Castelli F., Kurucz R.L.: 2004, arXiv: astro-ph/ $040508 \%$.

Galazutdinov G.A.: 2007, http://gazinur.com/DECHsoftware.html.

Kovtuykh V.V.: 2007, MNRAS 378, 617.

Kupka F., Piskunov N.E., Ryabchikova T.A., Stempels H.S., Weiss W.W.: 1999 A $\& A, \mathbf{1 3 8}, 119$.

Luck R.E.: 2014, AJ, 147, 137.

Luck R.E., Lambert D.L.: 2011, A, 142, 136.

Mel'nik A.M., Rautiainen P., Berdnikov L.N., Dambis A.K., Rastorguev A.S.: 2015, AN, 336, 70. 